\title{
The Second Virial Coefficient of Monodisperse Polystyrene in Cyclohexane below the Theta Point
}

\author{
Zhen Tong, Shigeyuki OHASHI, Yoshiyuki EINAGA, \\ and Hiroshi FuJITA \\ Department of Macormolecular Science, Osaka University, \\ Toyonaka, Osaka 560, Japan
}

(Received October 15, 1983)

\begin{abstract}
A light scattering study was made on monodisperse polystyrene + cyclohexane solutions in the range of molecular weight from 10000 to 500000 and that of temperature from $34.5^{\circ} \mathrm{C}(\theta)$ down to a value about $1{ }^{\circ} \mathrm{C}$ above the cloud point. The second virial coefficient $A_{2}$ was found to depend essentially on temperature only, at least in the range of molecular weight examined. This result is not in conformity with any current two-parameter theories for $A_{2}$ of polymer solutions.
\end{abstract}

KEY WORDS Polystyrene / Theta Point / Cloud Point / Light Scattering / Chemical Potential / Flory-Huggins Theory / Interaction Parameter / Second Virial Coefficient / Two-Parameter Theory /

Despite its basic importance in elucidating chain interactions in poor solvents, the second virial coefficient $A_{2}$ of polymer solutions at temperatures below the theta point $\theta$ remains less explored. Available theories on $A_{2}$ are still restricted to the two-parameter regime. ${ }^{1}$ It is doubtful whether such theories are adequately applicable to the temperature region below $\theta$, where recent investigations on the dimensions of isolated polymer chains have indicated the necessity of considering effects due to the ternary cluster integral. ${ }^{2-4}$ Except for Wolf and Adam's on polystyrene in tert-butylacetate, ${ }^{5}$ the available experimental data on $A_{2}$ below $\theta$ are very limited and fragmentary. ${ }^{6,7}$ As will be noted later, Wolf and Adam's finding does not conform to the prediction of the two-parameter regime in regard to molecular weight dependence. However, it seems too early to accept this as definitive, and more experiments must be undertaken before a general conclusion, if any, may be established on $A_{2}$ of polymer solutions below $\theta$.

In the course of our recent experimental evaluation of the Flory-Huggins interaction parameter, we had an opportunity of carrying out light scattering measurements to estimate $A_{2}$ on six monodisperse polystyrene samples in cyclohexane below $\theta{ }^{8}$ These samples ranged in molecular weight from
$10 \times 10^{3}$ to $500 \times 10^{3}$, and the measurement on each was effected down to a temperature as close to the cloud point as possible. The finding is presented in this paper.

\section{EXPERIMENTAL}

\section{Polystyrene Samples}

We started with five standard polystyrene samples F1, F2, F4, F20, F40 supplied by Toyo Soda Co., and one laboratory-made sample F7 (anionically polymerized by $\mathrm{Mr}$. $\mathrm{H}$. Watanabe of our department). These samples were subjected to precipitation and/or column fractionation to sharpen further their already sufficiently narrow molecular weight distributions. The main fractions of the respective series of fractions were chosen as the test samples. By GPC measurements, their ratios of weight-average to number-average molecular weights were found to be smaller than 1.03 . Hence, in the present data analysis, any of the samples was treated as completely monodisperse in molecular weight.

The molecular weight $M$ determined by light scattering in benzene at $25^{\circ} \mathrm{C}$ was 10000,21500 , $43600,181000,498000$, for the samples F1, F2, F4, F20, F40, respectively. GPC (chloroform, at $41^{\circ} \mathrm{C}$ ) 
combined with small angle laser light scattering gave an $M$ of 74000 for the sample F7.

\section{Preparation of Solutions}

Each polystyrene sample was dissolved in benzene and repeatedly filtered through a Millipore membrane until no light scattered by dust particles could be observed in the laser beam passing through the solution. The filtered solution was then freezedried in a vessel covered with a Millipore film. A proper amount of the dry polymer was weighed into a cylindrical light scattering cell and again thoroughly dried. The solvent cyclohexane was distilled and immediately poured into the cell in a dry bag filled with nitrogen. The cell was then sealed with a ground glass stopper. The solution was left standing overnight at a temperature between 30 and $40^{\circ} \mathrm{C}$ with occasional shaking. Complete dissolution of the polymer was confirmed by observing transparency of the solution, and also by the absence of unusual intensity fluctuation of light scattered from the solution at the beginning of each scattering measurement. The test solution was carefully treated to prevent it from phase separation prior to and during each measurement.

The polymer weight fraction $w$ was determined gravimetrically and changed to the volume fraction $\phi$ defined by

$$
\phi=\left[1+\left(v_{0} / v_{\mathrm{P}}\right)\left(w^{-1}-1\right)\right]^{-1}
$$

where $v_{0}$ and $v_{\mathrm{P}}$ are the specific volumes of the pure solvent and polymer, respectively. We used 1.2923 $\mathrm{cm}^{3} \mathrm{~g}^{-1}$ for $v_{0}$ and $0.9343 \mathrm{~cm}^{3} \mathrm{~g}^{-1}$ for $v_{\mathrm{P}}$ at any temperatures studied, though these refer to $25^{\circ} \mathrm{C}$. It should be noted that $\phi$ defined by eq 1 is the composition variable appearing in the framework of the Flory-Huggins theory, but it is not the conventional "true" volume fraction and may be regarded as a converted weight fraction. ${ }^{9}$

\section{Light Scattering Measurements}

Intensities of light scattered by polystyrene +

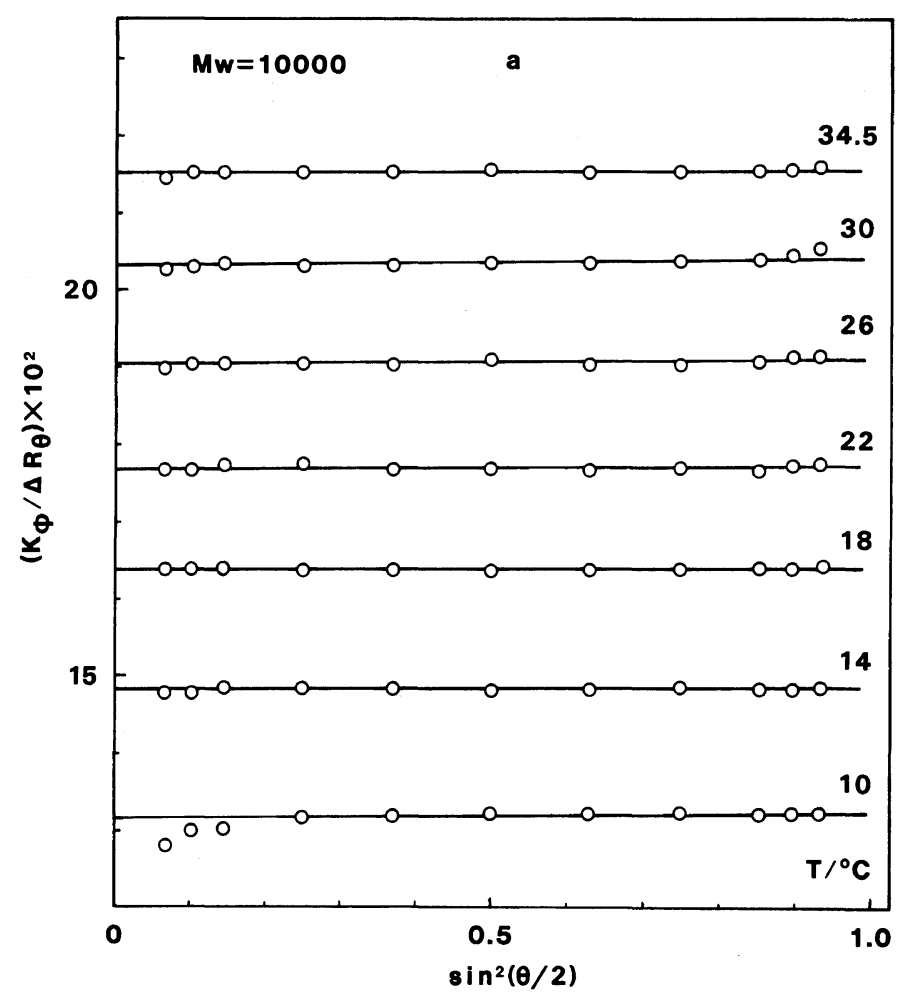

Figure 1a. Angular dependence of $K_{\phi} / \Delta R_{\theta}$ for the sample $F 1$ at the temperatures indicated and $\phi=0.0592$. 
cyclohexane solutions were measured at angles between 30 and $150^{\circ}$ in the temperature range from $34.5^{\circ} \mathrm{C}$ to a value about $1^{\circ} \mathrm{C}$ above the cloud point. Thus, the lowest temperature examined at a given concentration was sometimes lower than the critical temperature $T_{\mathrm{c}}$ for the solution. ${ }^{8}$ The temperature of each measurement was maintained within $0.05^{\circ} \mathrm{C}$.

Use was made of a Fica 50 light scattering photometer with polarized incident light of $546.1 \mathrm{~nm}$ in wavelength. Neutral density filters were inserted when scattering intensity was too high. Either a polystyrene + xylene solution or a glass block was used as the working standard to determine the transmittance of the filters and also to check the stability of the apparatus.

Under the reasonable assumption that the solution is incompressible and that no change in volume occurs on mixing, the excess Rayleigh ratio $\Delta R_{0}$ at zero scattering angle can be related to the derivative of the excess chemical potential $\Delta \mu_{0}$ of the solvent with respect to $\phi$ by

$$
K_{\phi} / \Delta R_{0}=-(1 / R T \phi)\left(\partial \Delta \mu_{0} / \partial \phi\right)
$$

with

$$
K_{\phi}=\left(4 \pi^{2} n^{2} / N_{\mathrm{A}} \lambda_{0}{ }^{4}\right)(\partial n / \partial \phi)^{2} / V_{0}
$$

Here, $R$ is the gas constant, $T$ the absolute temperature, $n$ the refractive index of the solution, $N_{\mathrm{A}}$ the Avogadro constant, $\lambda_{0}$ the wavelength of the incident light, $V_{0}$ the molar volume of the solvent $\left(V_{0}=v_{0} M_{0} ; M_{0}\right.$ the molecular weight of the solvent), and $\partial n / \partial \phi$ the refractive index increment. Values of $\partial n / \partial \phi$ at various temperatures and concentrations were calculated from Scholte's equation $^{10,11}$

$$
\partial n / \partial w=n_{0}+0.070 w+0.058 w^{2}
$$

with

$$
n_{0}=(693.7+2 T) \times 10^{-4}
$$

\section{RESULTS AND DISCUSSION}

\section{Derivative of Chemical Potential}

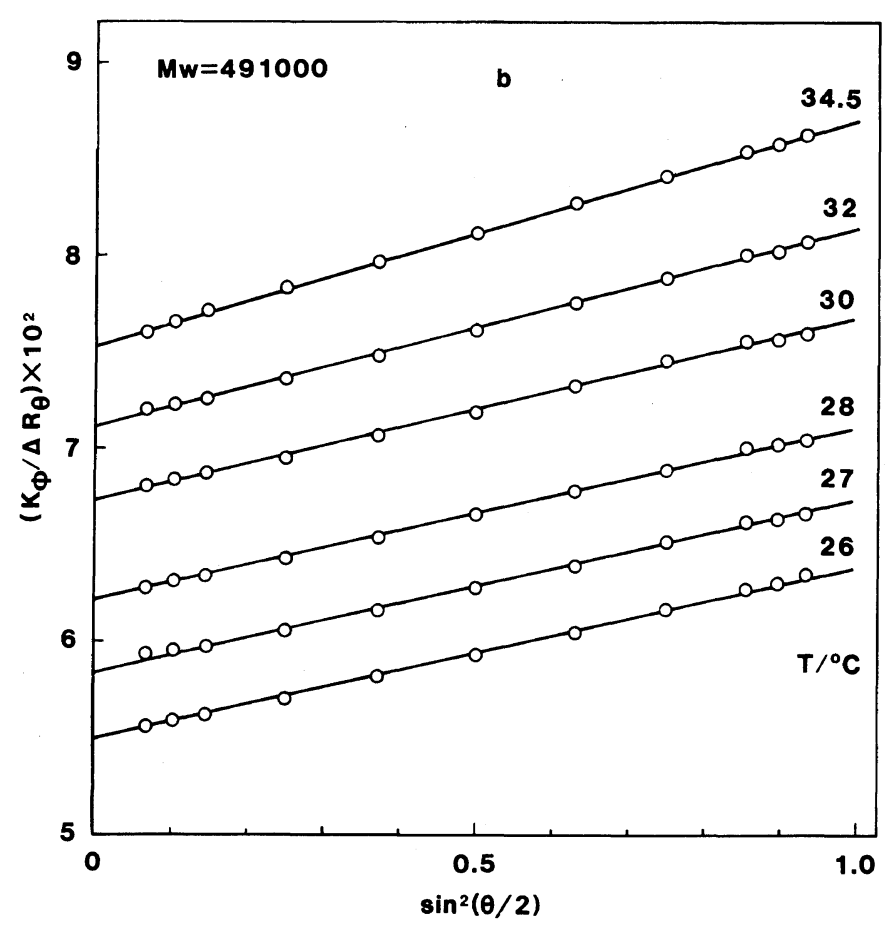

Figure 1b. Angular dependence of $K_{\phi} / \Delta R_{\theta}$ for the sample $\mathrm{F} 40$ at the temperatures indicated and $\phi=0.003188$. 


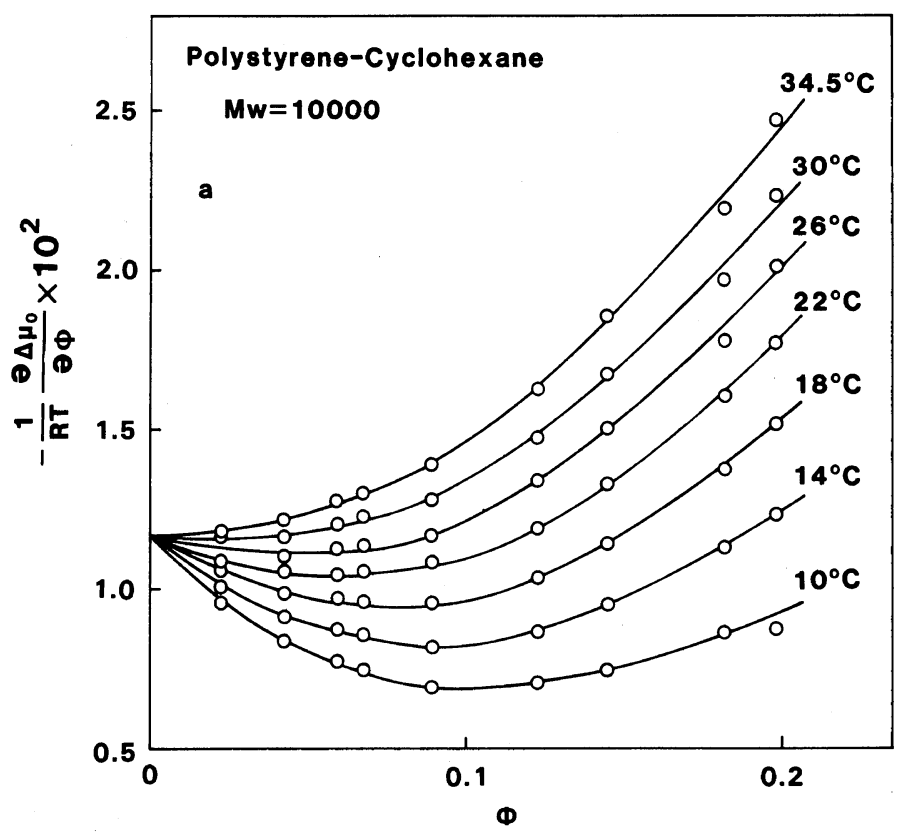

Figure 2a. Plots of $-(1 / R T)\left(\partial \Delta \mu_{0} / \partial \phi\right) v s$. $\phi$ for the sample $\mathrm{F} 1$ at the temperatures indicated. $\Delta \mu_{0}$ is the excess chemical potential of the solvent.

Figure 1 illustrates the angular dependence of $K_{\phi} / \Delta R_{\theta}$ for the samples $\mathrm{F} 1$ and $\mathrm{F} 40$. The data points at each temperature can be fitted closely by a straight line and extrapolated easily to the zero scattering angle to evaluate $K_{\phi} / \Delta R_{0}$ with sufficient precision. The lines at different temperatures are almost parallel, and those for the sample F1 are horizontal as expected from the low molecular weight of the sample.

Figure 2 depicts the $\phi$ dependence of $-(1 / R T)\left(\partial \Delta \mu_{0} / \partial \phi\right)$ for the samples $\mathrm{F} 1$ and F40 calculated from eq 2 . The values of $-(1 / R T)\left(\partial \Delta \mu_{0} / \partial \phi\right)$ at $34.5^{\circ} \mathrm{C}$ remain constant at relatively low $\phi$ and then increase with increasing $\phi$. At a lower temperature, $-(1 / R T)\left(\partial \Delta \mu_{0} / \partial \phi\right)$ decreases at first, passes through a minimum, and then increases as $\phi$ increases. These features of $-(1 / R T)\left(\partial \Delta \mu_{0} / \partial \phi\right) v s . \phi$ relations were common to the data on all the samples studied.

As can be seen from Figure 2, the data sets for different temperatures may be extrapolated to the same ordinate intercept. The inverse of this intercept equals the relative chain length $P$ of the sample defined by

$$
P=v_{\mathrm{P}} M / V_{0}
$$

The $P$ value found in this way was $86.0,375,1550$, 4220, 184, and 633 for F1, F4, F20, F40, F2, and $\mathrm{F} 7$, respectively. The molecular weights calculated from these $P$ values were $10000,43700,180000$, 491000,21400 , and 73700 , which agree closely with the values presented in the Experimental section. It should be noted that each sample gave an ordinate intercept or a $P$ value independent of $T$. This finding confirms that the solutions examined were completely free from intermolecular association or aggregation at any temperatures down to the vicinity of the cloud point.

\section{The Interaction Parameter}

Substitution of the Flory-Huggins equation for $\Delta \mu_{0}$ into eq 2 shows that the vaiable $Z$ defined by

$$
Z=\chi+(1 / 2)(\partial \chi / \partial \phi) \phi
$$

is represented by

$$
Z=\left[1 /(1-\phi)+1 / P \phi-K_{\phi} / \Delta R_{0}\right] / 2
$$

where $\chi$ is the Flory-Huggins interaction parameter. The right hand side of eq 8 consists only of 


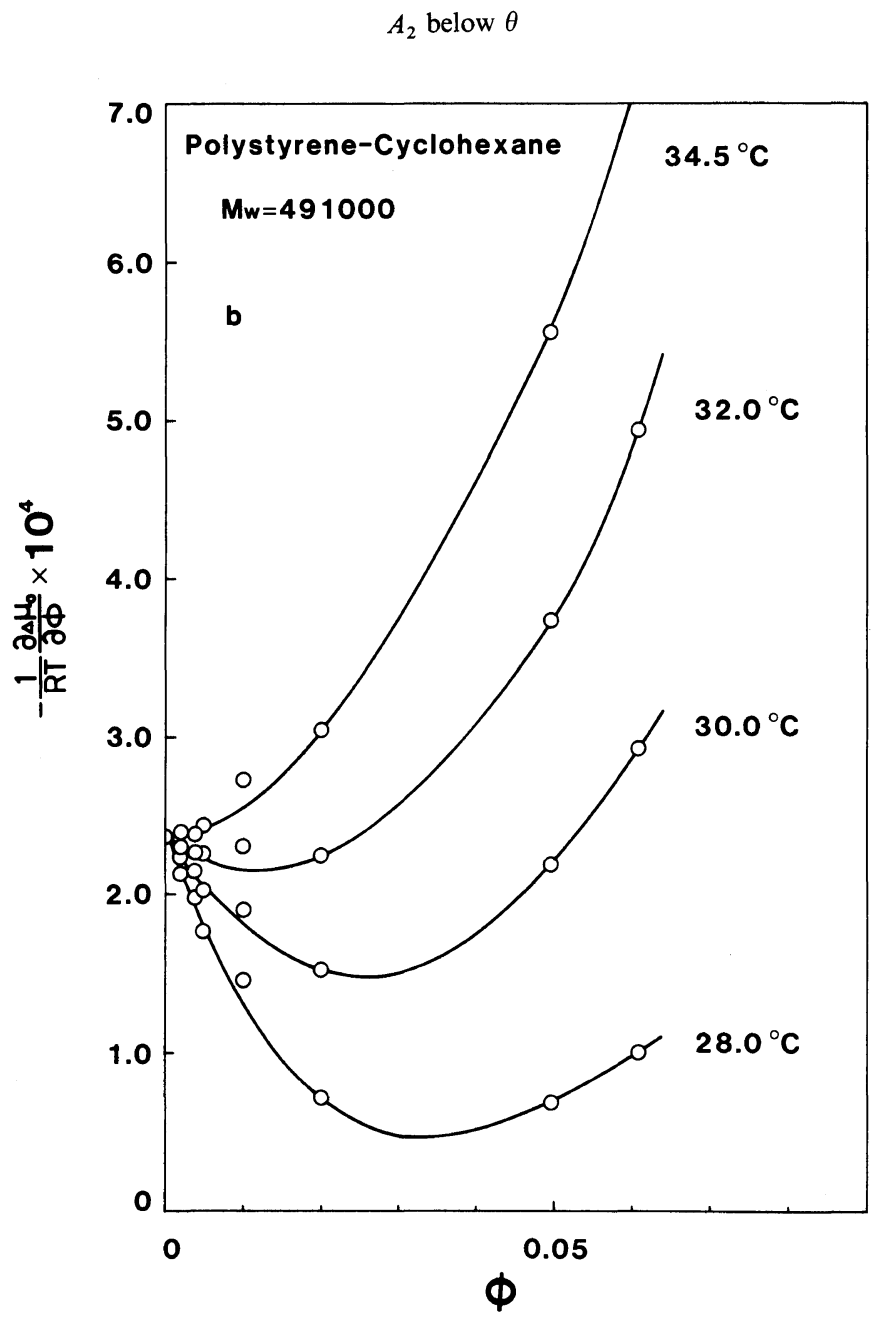

Figure 2b. Plots of $-(1 / R T)\left(\partial \Delta \mu_{0} / \partial \phi\right) v s . \phi$ for the sample F40 at the temperatures indicated.

experimentally measurable quantities. Figure 3 summarizes the $\phi$ dependence of $Z$ for the six samples studied. It should be noted that the data in this figure are confined to relatively low concentrations. The data points at $34.5^{\circ} \mathrm{C}$ for each sample can be fitted by a straight line, and the intercept is equal to or very close to 0.50 . Those at lower temperatures also follow a line straight or slightly bent upward, and it is not difficult to extrapolate $Z_{0}$, the value of $Z$ at zero concentration. From eq 7 it follows that $Z_{0}$ is equal to $\chi_{0}$, the value of $\chi$ at infinite dilution. The parameter $\chi_{0}$ is related to the second virial coefficient $A_{2}$ by

$$
A_{2}=\left(v_{\mathrm{p}}^{2} / V_{0}\right)\left(1 / 2-\chi_{0}\right)
$$

Thus, it is possible to evaluate $A_{2}$ as a function of $T$ and $M$ from the intercepts of the $Z v s . \phi$ plots in Figure 3.

\section{The Second Virial Coefficient}

Figure 4 shows $-A_{2}$ plotted against $T^{-1}$ for the six samples examined. This figure shows a striking fact that the data points for different $M$ virtually fall on a single curve. Allowing for the scatter of plotted points as due to experimental error, we may consider this fact to indicate that $A_{2}$ for monodisperse polystyrene in cyclohexane below $\theta$ depends essentially on temperature only. This conclusion does not conform to the prediction of any currently available two-parameter theories for $A_{2}$ of polymer solutions. ${ }^{1}$ For a clear-cut illustration of the discrepancy we here choose the latest version of these 


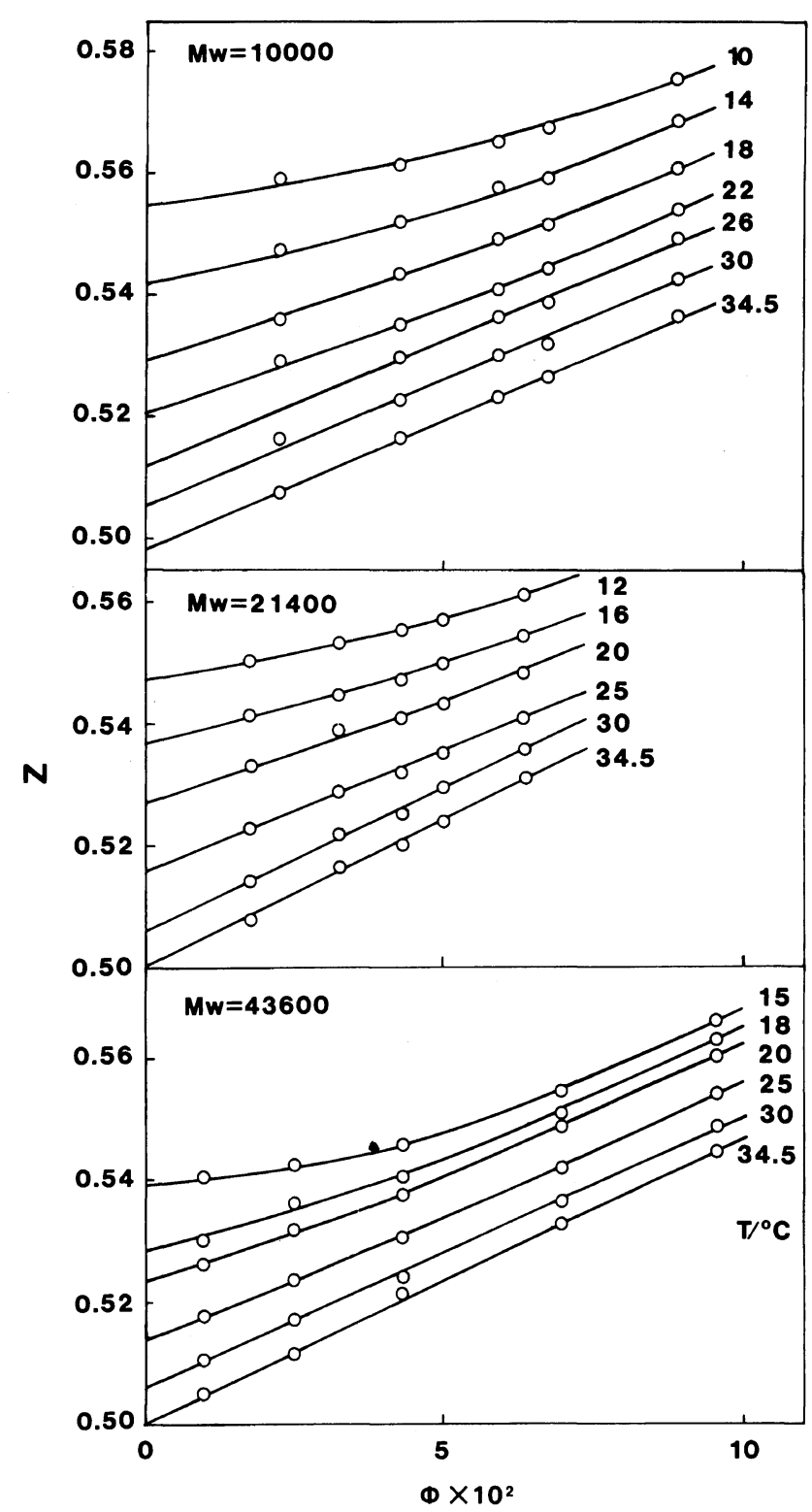

Figure 3a. Concentration dependence of $Z$ for the molecular weights and temperatures indicated. See eq 7 for the definition of $Z$.

theories, i.e., Tanaka and Solc's formulation, ${ }^{12}$ which gives

$$
A_{2}=\left(N_{\mathrm{A}} \beta / 2 M_{\mathrm{s}}^{2}\right) h(z)
$$

where

$$
h(z)=(1+6.856 z)^{-0.418}
$$

with

$$
z=\left(4 \pi\left\langle S^{2}\right\rangle_{0} / M\right)^{-3 / 2} M_{\mathrm{s}}^{-2} M^{1 / 2} \beta
$$

Here, $\beta$ is the binary cluster integral, $M_{\mathrm{s}}$ the molecular weight of a repeating unit, and $\left\langle S^{2}\right\rangle_{0}$ the unperturbed mean-square radius of gyration of the polymer. As far as the temperature not greatly removed from $\theta$ is concerned, as in the present study, $\beta$ may be put in the conventional form 


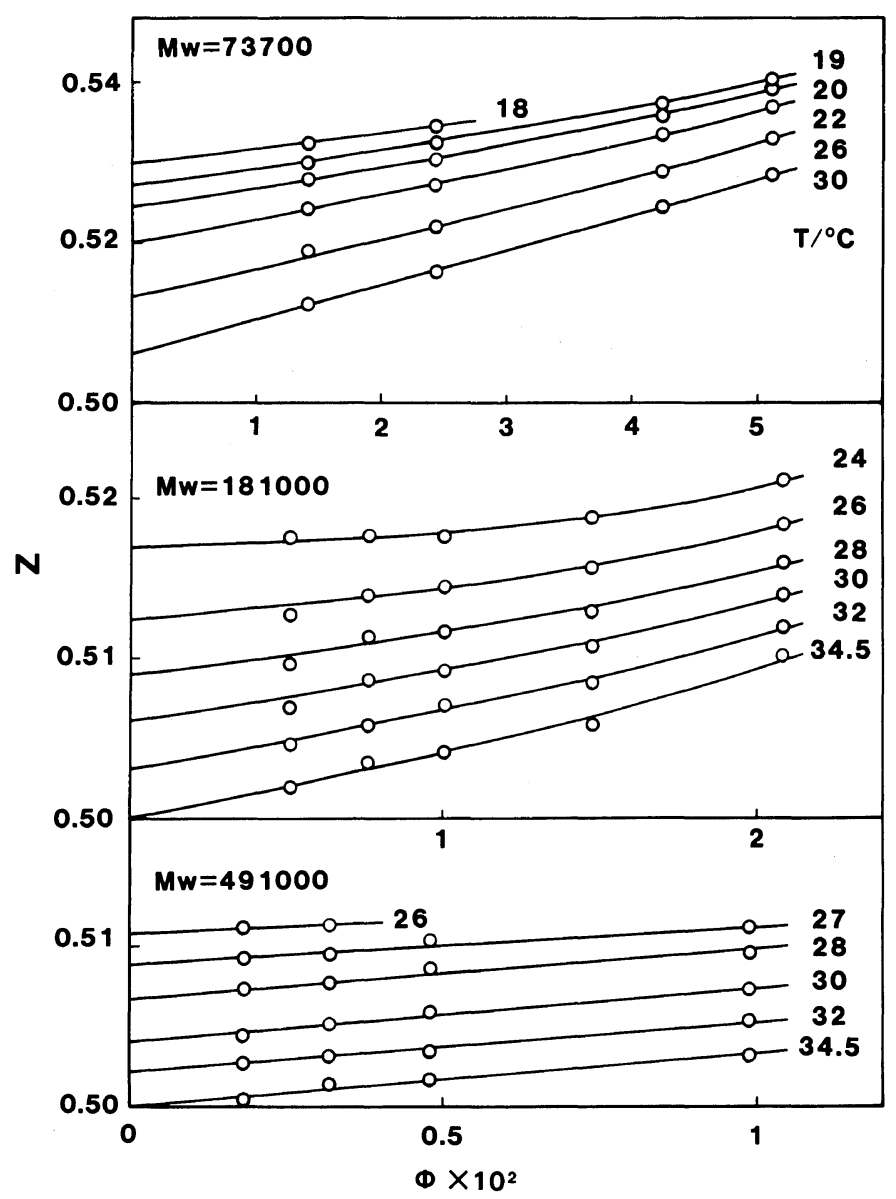

Figure 3b. Concentration dependence of $Z$ for the molecular weights and temperatures indicated. See eq 7 for the definition of $Z$.

$$
\beta=\beta_{0}(1-\theta / T)
$$

where $\beta_{0}$ is a constant characteristic of the polymer + solvent system under study. It is relevant to use Miyaki and Fujita's values ${ }^{13}$ of $\beta_{0}\left(7.2 \times 10^{-23} \mathrm{~cm}^{3}\right)$ and $\left\langle S^{2}\right\rangle_{0} / M\left(8.3 \times 10^{-18} \mathrm{~cm}^{2}\right)$ for computing $z$ and $\beta$ from eq 12 and 13. The solid lines in Figure 4 show the relations between $-A_{2}$ and $T^{-1}$ calculated in this way from eq 10 and 11 for the molecular weights of the samples studied.

In contrast to our experimental finding, these lines give a temperature dependence which varies distinctly with temperature, being stronger for higher molecular weight. Any other two-parameter theories, ${ }^{1}$ which are distinguishable only in the mathematical form of $h(z)$, yield the temperature and molecular weight dependence of $A_{2}$ substantially similar to what is illustrated in Figure 4 by the solid curves.

The following few remarks may help look at our finding in more detail.

(1) The calculated curves for different $M$ in Figure 4, and those from any other two-parameter theories, pass through $A_{2}=0$ at $T=\theta$ with a common tangent. Thus, in the regime of two-parameter theory, $A_{2}$ at temperatures very close to $\theta$ should be essentially independent of $M$. Our experimental data are seen to conform to this prediction. On the other hand, Wolf and Adam's data for polystyrene in tert-butylacetate ${ }^{5}$ give $A_{2} v s . T^{-1}$ curves which pass through $A_{2}=0$ at $T=\theta$ with an inclination which depends significantly on $M$. Thus, these 


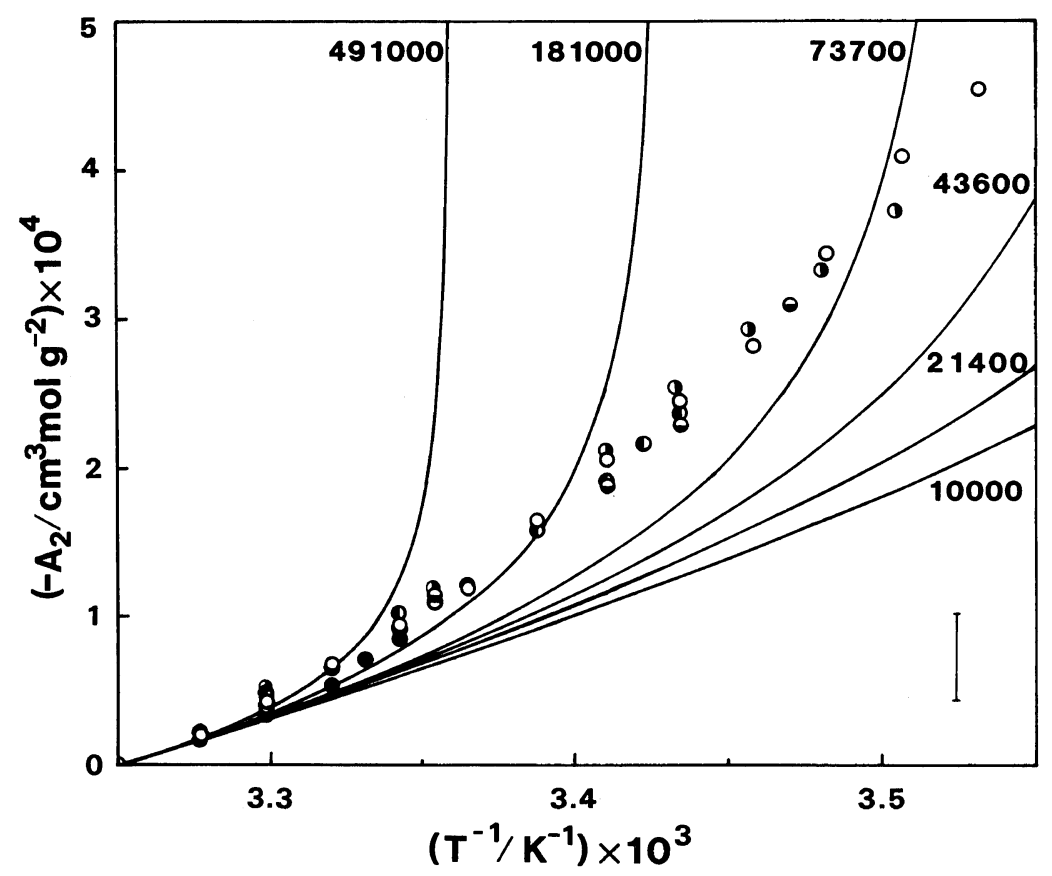

Figure 4. Negative second virial coefficient $-A_{2}$ plotted against $T^{-1}$ for six samples, whose molecular weights are as follows: $\bigcirc, 10000 ; \bigcirc, 21400 ; \Theta, 43600 ; \bigcirc, 73700 ; \ominus, 181000$, and $\odot, 491000$. Solid lines represent $-A_{2}$ calculated by Tanaka and Solc's equation for the $M$ values of the samples used. A probable experimental error of $\pm 2 \%$ in molecular weight determination causes $A_{2}$ to vary by $\pm 3 \times 10^{-5}$ $\mathrm{cm}^{3} \mathrm{molg}^{-2}$, which is indicated by a vertical bar in the figure. The inaccuracy of $A_{2}$ due to the extrapolations in Figures 1 and 3 is probably less than this.

curves cross with each other at $T=\theta$, giving larger $-A_{2}$ for a lower molecular weight at temperatures below $\theta$.

(2) For solubility reasons the actual measurement of $A_{2}$ below $\theta$ is impossible to extend beyond a certain temperature. This lower limit temperature approaches $\theta$ as the molecular weight of the sample becomes higher. Thus, it can be seen from Figure 4 that the set of data points for a given sample covers an increasingly narrower range of $T^{-1}$ as $M$ is increased. For example, the data for the highest molecular weight sample F40 range only from 34.5 $(\theta)$ to $26^{\circ} \mathrm{C}$. At present, we have no experimental means to see how $A_{2}$ of this sample varies at temperatures below $26^{\circ} \mathrm{C}$. No one can say any definitely that $A_{2}$ in such a temperature region should come close to the composite curve formed by the data points for lower molecular weight samples at the corresponding temperatures. Hence, it is legitimate to conclude from our experimental data that $A_{2}$ for monodisperse polystyrene in cyclohexane below $\theta$ appears to be independent of molecular weight, within the ranges of $T$ and $M$ studied.

(3) As explained above, any currently available two-parameter theories fail to predict this conclusion, and at present we have no idea allowing a molecular interpretation of our experimental finding. Except for Wolf and Adam's, ${ }^{5}$ published $A_{2}$ data below $\theta$ are too few and too fragmentary for a definite conclusion to be drawn on the temperature and molecular weight dependence of $A_{2}$. Thus, much remains to be investigated both theoretically and experimentally on $A_{2}$ of polymer solutions below the theta point.

Acknowledgment. The authors thank Dr. M. Fukuda of Toyo Soda Co., and Mr. H. Watanabe of our department for providing the polystyrene samples used in this study. 


\section{REFERENCES}

1. H. Yamakawa, "Modern Theory of Polymer Solutions," Harper \& Row, New York, 1971.

2. P. G. De Gennes, J. Phys., 36, L-55 (1975); ibid., 39, L-299 (1978).

3. T. Oyama and Y. Oono, J. Phys. Soc. Jpn., 44, 1348 (1977).

4. A. R. Khokhlov, J. Phys., 38, 845 (1977).

5. B. A. Wolf and H.-J. Adam, J. Chem. Phys., 75, 4121 (1981).
6. W. R. Krigbaum, J. Am. Chem. Soc., 76, 3758 (1954).

7. Y. Miyaki, Ph. D. Thesis, Osaka University, 1981.

8. Y. Einaga, S. Ohashi, Z. Tong, and H. Fujita, Macromolecules, to be published.

9. R. Koningsveld, Thesis, Leiden, 1967.

10. Th. G. Scholte, J. Polym. Sci., A-2, 8, 841 (1970).

11. Th. G. Scholte, Eur. Polym. J., 6, 1063 (1970).

12. G. Tanaka and K. Solc, Macromolecules, 15, 791 (1982).

13. Y. Miyaki and H. Fujita, Macromolecules, 14, 742 (1981). 